\title{
Information Sharing in Vehicular AdHoc Network
}

\author{
A. Rahim, Z.S. Khan, F.B. Muhaya, M. Sher, M.K. Khan
}

\author{
Aneel Rahim, Zeeshan Shafi Khan \\ 1. Prince Muqrin Chair for IT Security, \\ King Saud University, Saudi Arabia \\ 2. International Islamic University, Pakistan \\ E-mail: aneelrahim,zeeshanshafi@ksu.edu.sa \\ Fahad Bin Muhaya \\ Prince Muqrin Chair for IT Security, \\ King Saud University, Saudi Arabia \\ E-mail: fmuhaya@ksu.edu.sa

\section{Muhammad Sher \\ International Islamic University, Pakistan \\ E-mail: m.sher@iiu.edu.pk}

\section{Muhammad Khurram Khan} \\ Center of Excellence in Information Assurance, \\ King Saud University, Saudi Arabia \\ E-mail: mkhurram@ksu.edu.sa
}

\begin{abstract}
Relevance Technique broadcast the useful information and removes the redundant data. 802.11e protocol implementation has certain flaws and is not suitable for VANETs scenarios. Main issue in 802.11e protocol is internal sorting of packets, no priority mechanism within the queues and often lower priority traffic get more medium than high priority traffic. In this paper, the mathematical model of relevance scheme is enhanced so that it can consider the network control in real scenario by considering the impact of malicious node in network. Problems of 802.11e protocol can be resolved by making virtual queue at application level. We analyze the comparison of simple virtual queue with the over all impact of virtual queue and mathematical model. Similarly we compare the mathematical model with over all impact of virtual queue and modified mathematical model using NS-2 simulator.
\end{abstract}

Keywords: VANETs, Broadcast, 802.11e, Malicious

\section{Introduction}

Vehicle to Vehicle (V2V) communication enhances the safety of passenger and driver [1]. V2V communications is unreliable because of shadowing, Doppler shifts and multi-path fading. [2]

Security is an important concern in mobile adhoc network [3] [4] [5]. Attacks are easily launched on VANETs [6] because of high speed [7], no infrastructure and topology changes frequently [8] [9]. Several Security attacks are possible on safety application, which includes Denial of Service [10], Masquerade [11], fake information, false position information and ID disclosure [12]. Vehicular communication vulnerabilities are explained in [13] which include Jamming, Forgery, In-transit Traffic Tampering, Impersonation, Privacy Violation and On-board Tampering. Malicious data in VANET is because of distributed environment and unreliable components of data generation. [14] We in this paper resolve the problems of $802.11 \mathrm{e}$ protocol by making virtual queue at application and enhance the mathematical model of message benefit to consider 
the network traffic. We measure the global benefit in real scenario by considering the impact of malicious node. We also show the comparison of simple virtual queue with the over all impact of virtual queue and mathematical model. Similarly we compare simple mathematical model with over all impact of virtual queue and mathematical model. This paper is organized as follows: In section 2, we discuss relevance based approach, its characteristics and its implementation using cross layer, 802.11e and 802.11e with virtual queue. In section 3, proposed study and results are presented using NS-2. Lastly in section 4 conclusions is given.

\section{Related Work}

Relevance Technique disseminates the useful information and removes the redundant data [16]. Vehicle contains huge information that can't be shared to due the high speed of Vehicles. Technique which gives High priority traffic more medium as compare to low priority traffic is a suitable approach for VANETS. So relevance technique is the only option as it forward data according to its relevance.

Relevance Techniques based upon the calculation of relevance value of message and its distribution according to its priority $[15,16]$. Altruism, Application-oriented information differentiation, Controlled Unfairness is the basic characteristics of relevance based approach $[15,17,18]$.

\subsection{Cross Layer and 802.11 e Implementation}

Relevance Technique can be implemented through cross layer design or by 802.11 e protocol. In cross layer design, relevance value of every packet is measured at application layer and pass to link layer through packet header. Modified medium access control and interface queue broadcast the high priority traffic with help of application layer information. [15, 17]. 802.11e protocol implementation has certain flaws and is suitable for VANETs scenarios [18]. Main issue in $802.11 \mathrm{e}$ protocol is that it does not provide internal sorting of packets, no priority mechanism within the queues and performance of the network degrades as lower traffic some times get more medium than high priority traffic [15].

\section{$2.2 \quad 802.11$ Implementation with Virtual Queue}

802.11e protocol problems are overcome by adding four virtual queues at application level. Packets are sorted according to their priority and most importance messages are near the head of queues. Sorting is done by getting the current information from application layer and length of 802.11 e is set to be one. When $802.11 \mathrm{e}$ is empty, one high priority packet is moved from virtual queue to 802.11e queue. Packet in 802.11e queue does not mean that it always broadcast. If get a packet in virtual queue that has higher relevance than packet in $802.11 \mathrm{e}$ queue than we swap both the packets in order to achieve higher global benefit [19].

\subsection{Mathematically Model for Relevance Based Approach}

The mathematical model for relevance based approach is given below.

$$
\text { Message Benefit }=\frac{1}{\sum_{i=0}^{n} \alpha_{i}} * \sum_{i=0}^{n} \alpha_{i} * b_{i}(m, v, i)-------[18] \text {. }
$$

To determine the relevance value of message, Message (m), Vehicle (v), and Information (i) context parameters are used. The $\mathrm{N}$ parameters are computed with the help of application dependent function bi. The $\mathrm{N}$ parameters are then weighted with application dependent factors ai. In the end, all parameters are sum up and divided by the sum of all $a_{i}$. 


\subsection{Enhanced Mathematically Model for Relevance Based Approach}

As the existing model does not have the support for network control traffic. So network performance can improve by adding the network control in the mathematical model [20].

Enhanced Message Benefit $=\frac{1}{\sum_{i=0}^{n} \alpha_{i}} * \sum_{i=0}^{n} \alpha_{i} * b_{i}(m, v, i)+\sum_{i=0}^{n} P_{i}$

a) $\sum_{i=0}^{n} P_{i}=0$ if it is user traffic

Where as

$\sum_{i=0}^{n} P_{i}=1$ for Operational level network problem, $\sum_{i=0}^{n} P_{i}=2$ for Administrative level, $\sum_{i=0}^{n} P_{i}=$ 3 for Maintenance level

b) Message Benefit $=\sum_{i=0}^{n} P_{i}$ (for Network Traffic only)

If $0 \gtrless \sum_{i=0}^{n} P_{i} \lesseqgtr 3$ Then $\frac{1}{\sum_{i=0}^{n} \alpha_{i}} * \sum_{i=0}^{n} \alpha_{i} * b_{i}(m, v, i)=0$

User and Network traffic is assigned a value between zero and three, in order to handle them easily with four queues (Q0, Q1, Q2, and Q3) of 802.11e. High priority traffic is assigned Qo so that it can be forward before the packets in Q1, Q2 and Q3. Queues are assigned to user and network control traffic according to there relevance value. But in the existing approach there is no mechanism for priority for network control traffic. So the global benefit is enhanced by considering network traffic.

\section{Proposed Study and Results}

In this study we simulate the relevance based approach and calculate global benefit in ideal scenario that all nodes are doing their properly and there is no malicious node in the network. In the second scenario we consider the impact of malicious node and measure how much global benefit is decreased. The malicious nodes forward the relevant messages first but also inject some surplus information. In last scenario malicious node forward the surplus message first and ignore the relevant message. In order to validate the proposed study, we compare the performance of relevance based approach in real and ideal scenario with $802.11 \mathrm{e}$ protocol. NS2 , a network simulator [21], is used to simulate the behavior for relevance based approach in VANETs scenarios. We use Manhattan Mobility Model and traffic is generated by Generic Mobility Simulation Framework [22]. Vehicles are moving at a speed of $72 \mathrm{Km} / \mathrm{hr}$ to $108 \mathrm{Km} / \mathrm{hr}$ within an area of $3000 \mathrm{~m} \times 3000 \mathrm{~m}$ with transmission range of $300 \mathrm{~m}$. Performance of relevance based approach is measured by calculating the global benefit. 
Table 1: Simulation Parameters

\begin{tabular}{|l|l|}
\hline Parameters & Settings \\
\hline Channel & Wireless \\
\hline Vehicles & $50,100,150$ \\
\hline MAC protocol & $802.11 \mathrm{e}$ \\
\hline Time & $50 \mathrm{~s}$ \\
\hline Routing Protocol & DSDV \\
\hline
\end{tabular}

Network Simulator is used for the simulation and different parameter used in the following study is given in Table 1.

\subsection{Improvement due to Mathematical Model}

In this study we simulate enhanced mathematically model of message benefit shown above with existing relevance based approach. Figure 1(a) shows that 50 vehicles are moving at high speed and share safety and comfort information with each other. Relevance based approach consider only user traffic and ignore network traffic. So its global benefit can be improved by improving the mathematical model. We now evaluate the performance of relevance based approach by adding the network control parameter in the existing formula. Figure 1(a) shows the global benefit with enhanced relevance based approach. It is clear from figure 1(a) that global benefit is low by using existing relevance based approach because network control traffic set lower priority and get less bandwidth than user traffic. So lower priority traffic can get more bandwidth than higher priority traffic. That's why the global benefit is improved by adding the network parameter in relevance based approach.
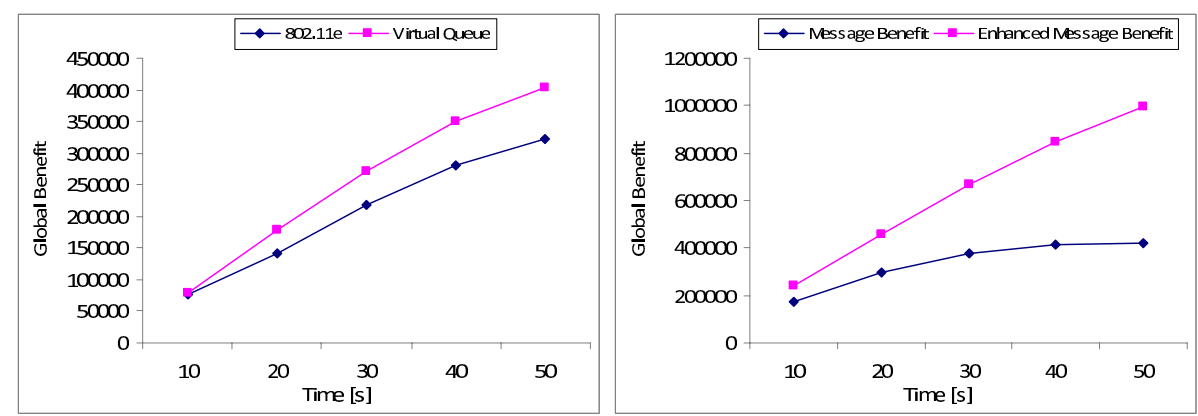

Figure 1: (a) Improvement due to mathematically (b) improvement due to virtual queue

\subsection{Improvement due to Virtual Queue}

Figure 1(b) shows simple 802.11e and virtual queue with $802.11 \mathrm{e}$, safety messages and route messages are forwarded by vehicles. In this study 150 vehicles exchanging information with each other. In simple $802.11 \mathrm{e}$, there is no mechanism of priority assignment. This problem is resolved by virtual queue. So its global benefit is greater than simple 802.11 e because it does not allow lower priority traffic to get more medium than higher priority traffic.

\subsection{Improvement due to Virtual Queue and Mathematically Model}

First we check the improvement due mathematical model and virtual queue separately but now we consider the impact of both on the global benefit of network. Figure 2 shows that global benefit of existing and enhance relevance based approach due to virtual queue and mathematically 
model. Enhanced relevance based approach has higher global benefit because it resolves the problem of priority mechanism and ignorance of network control traffic.

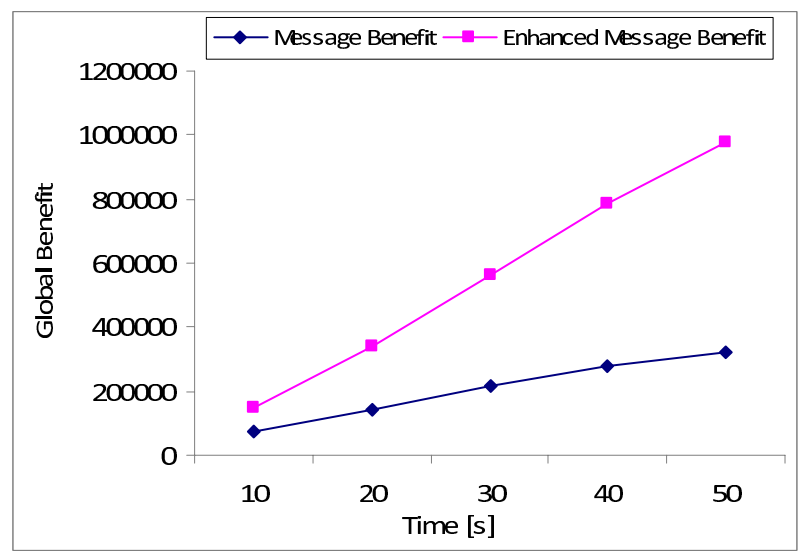

Figure 2: Improvement due to virtual queue and Mathematically model

\subsection{Comparison}

This study shows the comparison of simple virtual queue with the over all impact of virtual queue and mathematical model. Similarly we compare simple mathematical model with over all impact of virtual queue and mathematical model. Fig 3(a) shows the global benefit due to Message Benefit (MB), enhance message benefit (EMB) and virtual queue + EMB. It is clear from figure that global benefit by using virtual queue + EMB is greater than simple EMB because within a queue a there is no priority mechanism available.

Fig 3(b) shows the global benefit due to 802.11e, Virtual Queue and EMB + virtual queue. It is clear from figure that global benefit by using EMB + virtual queue is greater than $802.11 \mathrm{e}$ and simple queue because in simple queue we don't have discriminate between user traffic and network traffic.
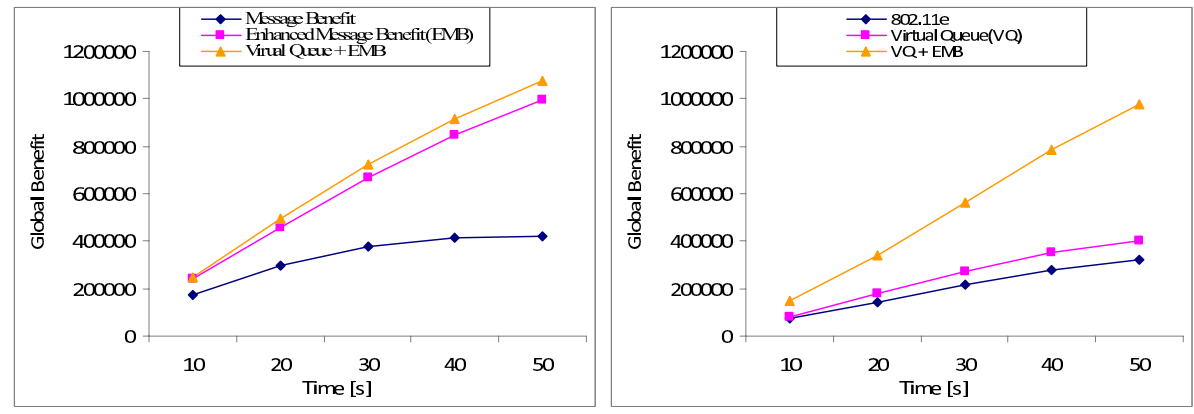

Figure 3: (a)Comparison of mathematically model with both virtual queue and mathematical model (b) Comparison of virtual queue with both mathematical models

\subsection{Impact of Malicious node}

In this study we consider the impact of malicious node on EMB, Virtual Queue and both (EMB + virtual queue).Figure 4(a) shows that 50 vehicles are moving at high speed and share safety and comfort information with each other. First we simulate the MB and EMB in ideal 
scenario that no malicious node exists and all nodes try to improve the benefit of network rather than their own benefit. After that we simulate EMB in real scenario that malicious exist and damage the performance of the network. Figure 4(a) shows that global benefit of EMB in real scenario lies between the EMB MB in ideal scenario.

Figure 4(b) shows that 150 vehicles exchanging information with each other. First we simulate the $802.11 \mathrm{e}$ and virtual queue in ideal scenario that no malicious node exists and all nodes try to improve the benefit of network rather than their own benefit. After that we simulate Virtual queue in real scenario that malicious exist and damage the performance of the network. Figure 4(b) shows that global benefit of EMB in real scenario lies below than 802.11e and Virtual Queue in ideal scenario.

Figure 5 shows that 150 vehicles are moving at high speed and share safety and comfort information with each other. First we simulate the MB and VQ + EMB in ideal scenario that no malicious node exists and all nodes try to improve the benefit of network rather than their own benefit. After that we simulate $\mathrm{VQ}+\mathrm{EMB}$ in real scenario that malicious exist and damage the performance of the network. Figure 5 shows that global benefit EMB + VQ in real scenario lies between the EMB $+\mathrm{VQ}$ and $\mathrm{MB}$ in ideal scenario.
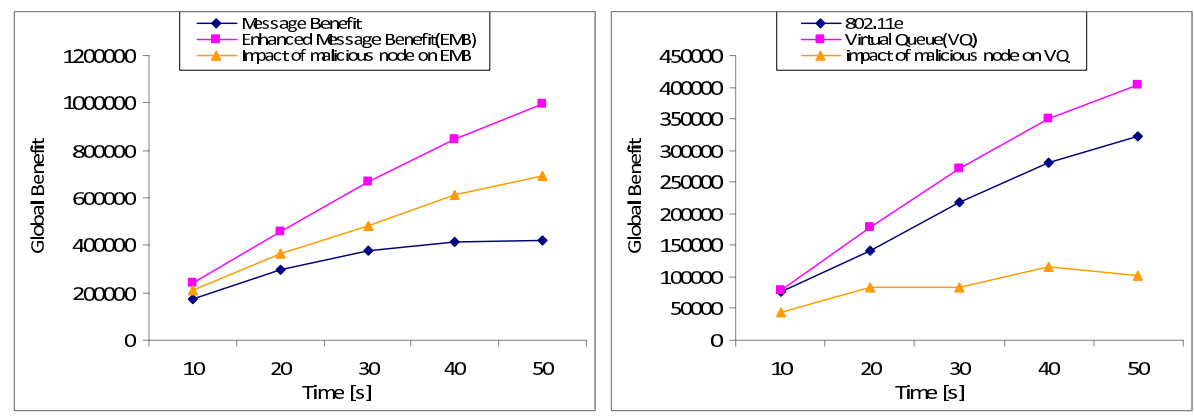

Figure 4: (a) Impact of malicious node on EMB (b)Impact of malicious node on Virtual Queue (VQ)

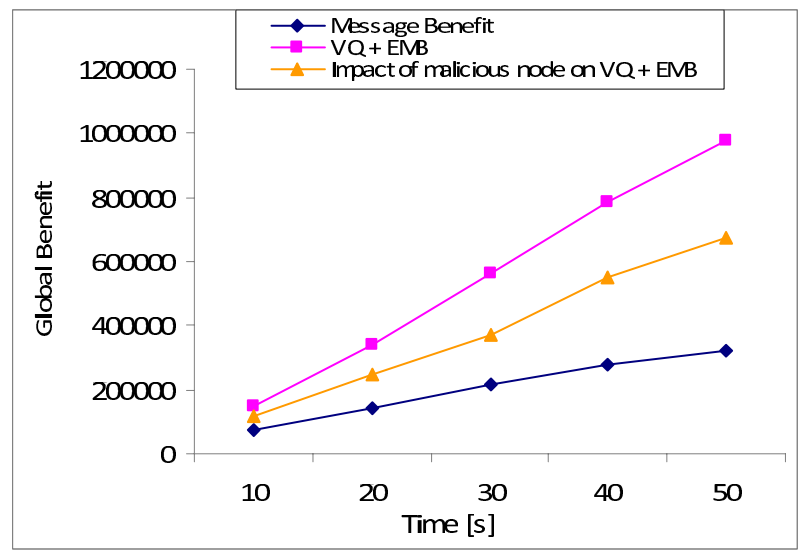

Figure 5: Impact of malicious node on Enhanced Message Benefit and Virtual Queue

\section{Conclusion}

Relevance scheme rely on intermediate node for communication so it consider there is no selfish node exist in network. However it is not possible in real scenario. We in this paper simulate 
the relevance based approach using 802.11e, virtual queue with 802.11e and enhance message benefit in real and ideal scenario. Simulation results shows that global benefit is improved by using virtual queue with enhance mathematical model.

\section{Acknowledgments}

This research is supported by the Prince Muqrin Chair (PMC) for IT Security at King Saud University, Riyadh, Saudi Arabia.

\section{Bibliography}

[1] Y. Wu, L. Yang, G. Wu, J. Guo, An Improved Coded Repetition Scheme for Safety Messaging in VANETs, IEEE, 2009.

[2] R. K. Shrestha, S. Moh, I. Chung, D. Choi, Vertex-Based Multihop Vehicle-to-Infrastructure Routing for Vehicular Ad Hoc Networks, IEEE, Proceedings of the 43rd Hawaii International Conference on System Sciences 2010.

[3] H. Kumar, , R.K Singla,., S. Malhotra, Issues and Trends in AutoConfiguration of IP Address in MANET, International Journal of Computers Communications and Control, Volume:3, Supplement: Suppl. S, pp. 353-357, 2008.

[4] M. A Rajan, M. G Chandra, L. C. Reddy, P. Hiremath, Concepts of Graph Theory Relevant to Ad-hoc Networks, International Journal of Computers Communications and Control, Volume:3, Supplement: Suppl. S, pp. 465-469, 2008.

[5] J. Sun, C. Zhang, Y. Zhang, Y. Fang, An Identity-Based Security System For User Privacy in Vehicular Ad Hoc Networks, IEEE Transactions On Parallel And Distributed Systems, 2010

[6] S. Dietzel, E. Schoch, B. Konings, M. Weber, Resilient Secure Aggregation for Vehicular Networks, IEEE Network, vol 24 pp 26-31,2010

[7] I. Jang, W. Choi, H. Lim, A Forwarding Protocol with Relay Acknowledgement for Vehicular Ad-Hoc Networks, IEEE 2008.

[8] H. L. Nguyen, U. T. Nguyen, Study of Different Types of Attacks on Multicast in Mobile Ad Hoc Networks, International Conference on Mobile Communications and Learning Technologies, IEEE , 2006.

[9] S. Mao, S. Lin, S. S. Panwar, Y. Wang, E. Celebi, Video Transport Over Ad Hoc Networks: Multistream Coding With Multipath Transport, IEEE Journal on Selected areas in Communications, vol. 21, no. 10, December 2003

[10] B. R. Moyers, J. P. Dunning, R. C. Marchany, J. G. Tront, Effects of Wi-Fi and Bluetooth Battery Exhaustion Attacks on Mobile Devices, Proceedings of the 43rd Hawaii International Conference on System Sciences, 2010.

[11] K.A. Bakar, B. S. Doherty, Evaluation of the Recorded State Mechanism for Protecting Agent Integrity Against Malicious Hosts, International Journal of Computers Communications and Control, Vol.3, No.1, pp. 60-68, 2008

[12] M. Raya, J-P. Hubaux. The security of vehicular ad hoc networks. In Workshop on Security in Ad hoc and Sensor Networks (SASN), 2005. 
[13] M. Raya, P. Papadimitratos,J.-P. Hubaux, Securing Vehicular Communications, In IEEE Wireless Communications Magazine, Special Issue on Inter-Vehicular Communications, October 2006.

[14] K. Sha,, S. Wang, W. Shi, RD4: Role-Differentiated Cooperative Deceptive Data Detection and Filtering in VANETs, IEEE Transactions On Vehicular Technology, vol. 59, no. 3, March 2010 .

[15] T. Kosch, C. J. Adler, S. Eichler, C. Schroth, M. Strassberger, The scalability problem of vehicular ad hoc networks and how to solve it, IEEE Wireless Communications, October 2006 .

[16] C. Adler, S. Eichler, T. Kosch, C. Schroth, M. Strassberger, Self-organized and ContextAdaptive Information Diffusion in Vehicular Ad Hoc Networks, 3rd International Symposium on Wireless Communication Systems, 2006.

[17] S. Eichler, C. Schroth, T. Kosch, M. Strassberger, Strategies for context-adaptive message dissemination in vehicular ad hoc networks, Second International Workshop on Vehicle-toVehicle Communications, July 2006.

[18] C. Schroth, R. Eigner, S. Eichler, M. Strassberger, A Framework for Network Utility Maximization in VANETs, ACM International Conference on Mobile Computing and Networking, USA September 29, 2006.

[19] A. Rahim, M. Yasin, I. Ahmad, Z. S. Khan, M. Sher, Relevance Based Approach with Virtual Queue Using 802.11e protocol for Vehicular Adhoc Networks, 2nd International conference on Computer, Control and Communication, Karachi, 14 Feb 2009.

[20] A. Rahim, F. B. Muhaya, Z. S. Khan, M.A. Ansari, M. Sher, Enhance Relevance based approach for Network Control Relevance, Accepted in Infomatica Journal ISSN: 0350-5596.

[21] Network Simulator, ns2 http://www.isi.edu/nsnam/ns

[22] R. Baumann, F. Legendre, P. Sommer, Generic Mobility Simulation Framework (GMSF), ACM MobilityModels'08, Hong Kong SAR, China, May 26, 2008 